\title{
Long-term Radio Behaviour of the X-ray Binary Circinus X-1*
}

\author{
Valeriu Tudose $\dagger$ \\ Astronomical Institute, University of Amsterdam \\ E-mail: vtudose@science.uva.nl
}

\section{Rob Fender}

University of Southampton

E-mail: rpfephys.soton.ac.uk

\section{Tasso Tzioumis}

Australia Telescope National Facility

E-mail: tasso.tzioumisecsiro.au

\section{Ralph Spencer}

Jodrell Bank Observatory

E-mail: res@jb.man.ac.uk

Circinus X-1 is a neutron star X-ray binary system with an interesting and at times puzzling behaviour over a broad range of frequencies, specifically in the $\mathrm{X}$-ray and radio bands. The system seems to harbour the most relativistic outflow (likely oriented close to the line of sight) observed so far within the Milky Way. It lies within a radio synchrotron nebula and has variable radio flux densities at $\mathrm{cm}$ wavelengths. The radio flares associated to the orbital phase zero reached up to 1 Jy in the late '70s, then have been observed at the tens of mJy level until recently; in 2007 January, Circinus X-1 seemed to have entered a very active radio flaring state. Here we present a sample of the 4.8 and $8.6 \mathrm{GHz}$ radio observations made with the Australia Telescope Compact Array, covering 10 years time period. The entire data set comprised 41 epochs, unequally spread in time between 1996 and 2006. We investigate the long-term changes in the brightness, morphology and spectrum of the radio structures. We have detected linear polarisation in a third of the epochs and a good case for Faraday rotation in one epoch. The analysis reveals structural changes in the radio emission at time scales as short as days. Clear evidence for a counter-jet was found in a few epochs.

Bursts, Pulses and Flickering: wide-field monitoring of the dynamic radio sky 12-15 June 2007

Kerastari, Tripolis, Greece

\footnotetext{
*A more detailed version of this contribution to be submitted to MNRAS.

† Speaker.
} 


\section{Introduction}

Circinus X-1 is one of the most exotic X-ray binary systems. The detection of type I Xray bursts [1], 2] and twin $\mathrm{kHz}$ quasi-periodic oscillations in the $\mathrm{X}$-ray power spectra [⿰]𠃌 strongly suggests that the compact object in the system is a neutron star. The system exhibits periodic flares $(\mathrm{P} \sim 16.6 \mathrm{~d})$ observed in $\mathrm{X}$-ray [4], infrared [5, 6] and radio [7. 8] bands, which are interpreted as enhanced accretion near the periastron passage. Circinus X-1 is associated with an arcmin scale synchrotron nebula [9, 10] which is probably powered by the jet originating close to the binary system [11, 12]. The jet is observed in radio band at arcmin as well as arcsec scales and was recently tentatively detected in X-ray band by Chandra [13]. Moreover, observations at $\mathrm{cm}$ wavelengths offered evidence for the presence of a relativistic outflow aligned very close to the line of sight [14].

\section{Observations}

The X-ray binary Circinus X-1 was observed for 41 epochs during a time interval of almost 10 years, between 1996 and 2006, simultaneously at 4.8 and 8.6 GHz with the Australia Telescope Compact Array (ATCA). The observations were carried out at different spatial resolutions (most of the data taken in the $6 \mathrm{~A}$ and $6 \mathrm{C}$ array configurations, but the $6 \mathrm{~B}, 6 \mathrm{D}, 6 \mathrm{~F}$ and $1.5 \mathrm{D}$ set-ups are also present). To assure an homogeneous analysis, in obtaining the final radio images we have used the same restoring beam (size and orientation) for the entire data set at each of the two observed frequencies. In the one decade of monitoring we swept the entire orbit of the system, even though more often than not only once for a given orbital phase, and gaps in the coverage are present.

\section{Secular evolution}

Fig. 1 shows the "secular" light curves of Circinus X-1 in radio and X-ray bands, between 1996-2006. The averaged X-ray output in the $2-10 \mathrm{keV}$ band was constant around 75 counts s$^{-1}$ up to the beginning of 2000 when it started to decrease relatively fast reaching only a few tens of counts $\mathrm{s}^{-1}$ by the end of 2003. The radio fluxes plotted are determined in the image-plane and are total flux densities, in the sense that they contain both the contribution of the core and other radio emitting regions (jet features, knots) pertaining to the system. The radio data offer a totally different picture than in the 1970-1980s when the flares usually reached flux density levels of up to 1 Jy [7, 8]. From 1996 till 2006 the outbursts only peaked at a few tens of mJy. It is true that we only observed a few times close to the orbital phase 0.0 , but the flux densities measured at other phases also further support the evidence that indeed dramatic changes took place in the system since the 1980s. The true nature of these changes is still unclear.

At a smaller temporal scale, whether the decrease in the X-ray output observed starting with 2000 can be correlated with a change in the radio behaviour is hard to asses with confidence. It does seem that the radio flux density levels became lower by comparison with the ones observed at similar orbital phases before, however the limited number of observations cannot exclude the possibility of this being an observational bias. In addition, the flare observed in 2005 June had the highest flux density in the whole data set, but this might just as well be associated with a seemingly increase in the radio activity of Circinus X-1 as was observed in the last few years [18, 19, 20]. 


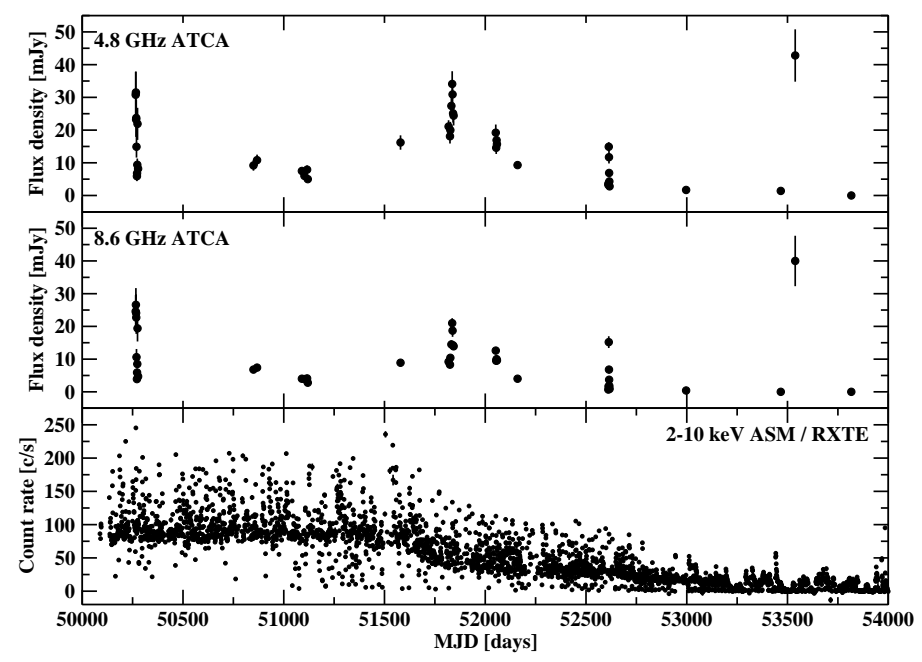

Figure 1: Secular evolution of Circinus X-1. Top and Middle: Variation of the total flux density between 1996-2006 at 4.8 and 8.6 GHz from the ATCA radio data. Bottom: 2-10 keV X-ray light curve over the same time interval from ASM/RXTE.

Fig. 2 shows a sample (10 out of 41 epochs) of radio images of Circinus X-1 at 4.8 and 8.6 GHz. As already previously noted (e.g. [17]) a jet-like structure is present towards the SE and is interpreted as the approaching jet. In the same frame-work, in a few cases (e.g. 2000 October 20/21) the receding jet was also identified. Some of the observations close to phase 0.0 (e.g. 2005 June 17) show a significant different orientation of the jet axis than the "normal" NW-SE direction. This is very likely an artifact resulting from variability of the source during the aperture synthesis. Test maps made in these cases with uv-plane selected data outside the instants of the outbursts show that the bulk radio emission has a NW-SE orientation.

The observations show changes in the morphology and brightness of the radio features on timescales of days. Individual, well defined emitting "blobs" are seen as far as 10 arcsec from the core, along a NW-SE direction. The limited amount of data (mainly the lack of enough closely spaced observations) didn't allow the measurement of the proper motion of these features. However, by studying the time delay between the successive increase in their brightness after a flaring event in the core, we were able to conclude that the data is compatible with the existence of a relativistic flow in the system.

\section{Proper motion}

The Circinus X-1 complex is on the plane of the sky just $\sim 10$ arcmin away from the boundary of the supernova remnant SNR G321.9-0.3. This offered circumstantial evidence for the so called "runaway binary" scenario in which the binary system and the supernova remnant are physically associated [15]. However, optical observations taken 8.6 years apart [16] placed a $3 \sigma$ upper limit

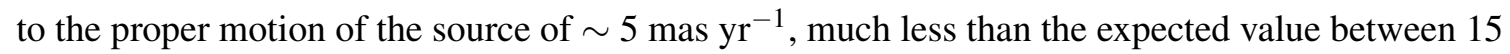

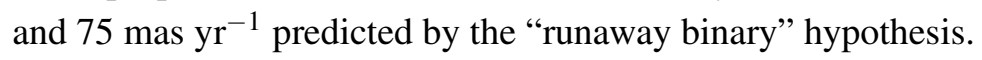



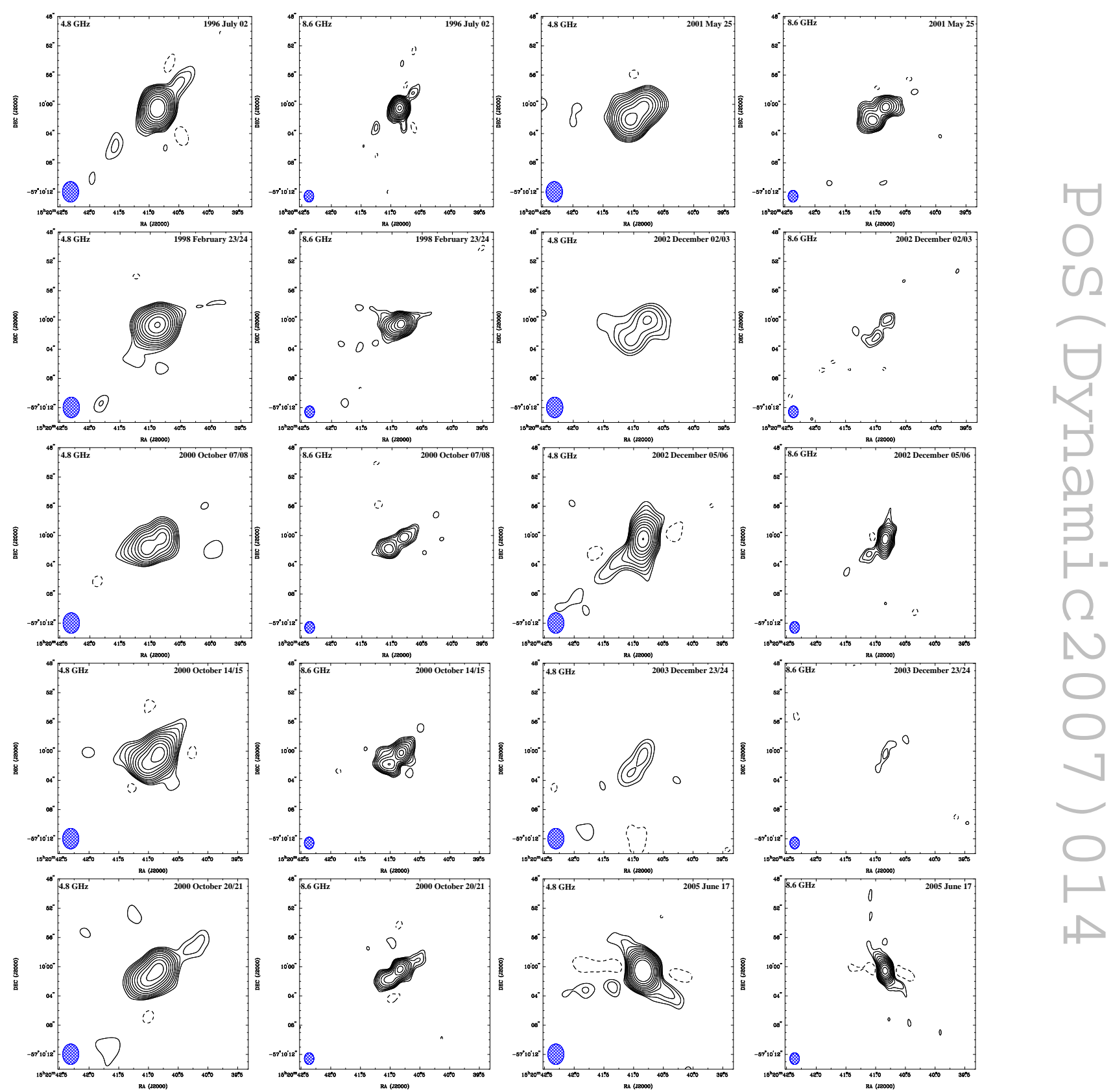

Figure 2: A sample of radio images (natural weightings) of Circinus X-1 at 4.8 and $8.6 \mathrm{GHz}$. The contour lines are at $-2.8,2.8,4,5.6,8,11,16,23,32,45,64,90 \times$ the rms noise at each epoch. 

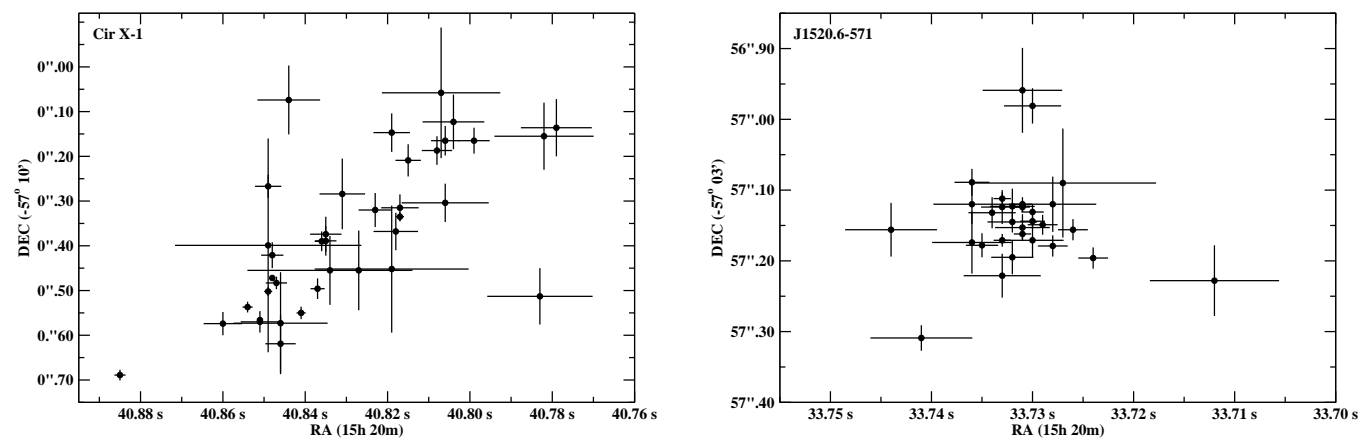

Figure 3: Positions of the core of Circinus X-1 (left) and J1520-571 (right) between 1996-2006. The coordinates were obtained via image plane fitting of the $8.6 \mathrm{GHz}$ data. For an easier comparison the error bars do not contain the systematic errors associated with the position of the phase-referencing calibrator.

In Fig. 3 (left) we plot the positions of the core of Circinus X-1 between 1996-2006 as determined from the $8.6 \mathrm{GHz}$ data. The diagram shows an apparent tendency of the points to align along a NW-SE direction. However, the position of the object "jumps" randomly from one epoch to another, with no preferential direction. To test if this could be explained by errors in the phasereferencing process, we used a compact source $\sim 7$ arcmin away from Circinus X-1, designated J1520.6-571 by [17], which was observed during the runs. After phase-referencing it with respect to the same calibrator as for Circinus X-1 we fitted the data in the image plane. The resulting positions are reported in Fig. 3 (right). The lack in this case of a similar trend in the distribution of positions as observed for Circinus X-1 strongly suggests that the phase-referencing process is not responsible for the tendency (or, more conservatively, is not the dominant factor). Instead, given the magnitude of the errors and the fact that the bulk of the radio emission in Circinus X-1 is oriented on a NW-SE direction (the jet axis), it is very likely that this tendency is an artifact of the fitting process. Therefore, our roughly estimated upper limit to the proper motion of Circinus X-1

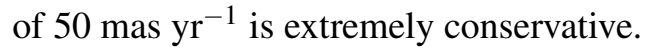

The errors in the position of the core are dominated at most of the epochs by the positional error of the phase-referencing calibrator (reported to be between 100 and 250 mas). Future longterm observations of Circinus X-1 combined with a better knowledge of the systematic errors due to the calibrator will enable to further constrain the proper motion down to, and ultimately even better than the presently available optical limits.

\section{Polarisation}

Out of 41 observational runs, polarisation was detected in 13 epochs at $4.8 \mathrm{GHz}$ and in one epoch at $8.6 \mathrm{GHz}$. Fig. 4 shows a sample of the polarisation maps of Circinus X-1. The fractional linear polarisation was of a few percent in the entire data set, from 1996 until 2006. It is also worth mentioning that there is no significant evidence that the orientation of the electric field vectors changed during the period covered by our observations. 

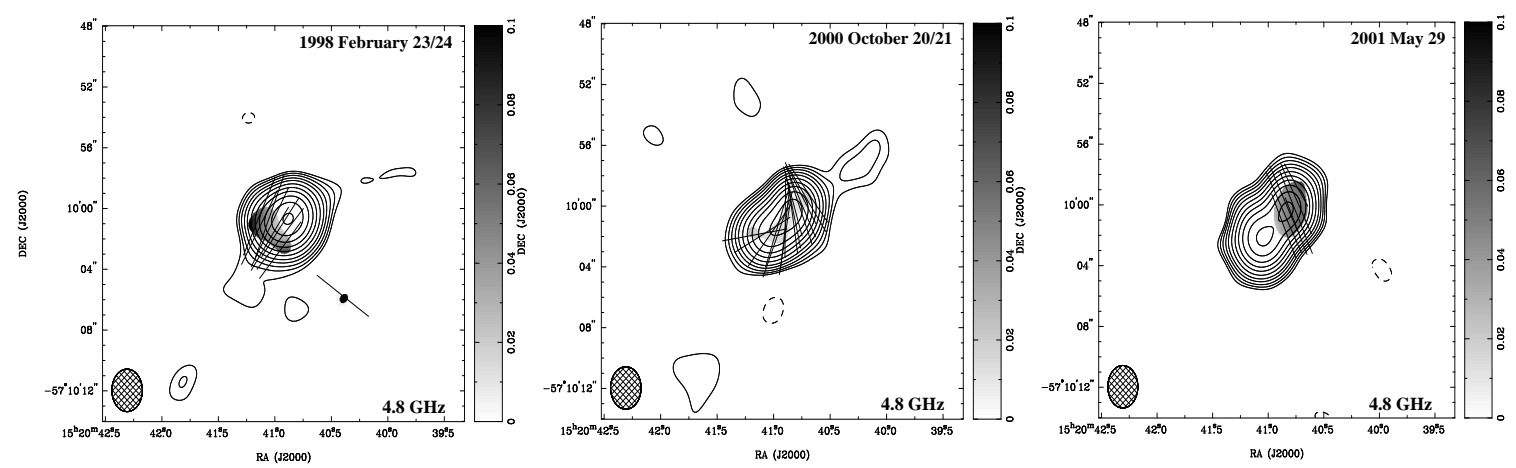

Figure 4: A sample of radio polarisation maps of Circinus X-1 at $4.8 \mathrm{GHz}$. The contour lines are at -2.8 , $2.8,4,5.6,8,11,16,23,32,45,64,90 \times$ the rms noise at each epoch. The thick, straight lines correspond to the orientation of the electric vector position angles. Superimposed is the gray-scale map of the fractional linear polarisation.

Faraday rotation (i.e. rotation of the observed plane of polarisation due to propagation of radiation through magnetized plasma) was detected close to the core of the system in one epoch at a level of $-175 \pm 30 \mathrm{rad} \mathrm{m}^{-2}$. It is not clear yet what is its origin. Preliminary evidence seems to point towards an intrinsic nature. And if the galactic Faraday rotation measure in the direction of Circinus X-1 is somewhere between -50 and $50 \mathrm{rad} \mathrm{m}^{-2}$ as some circumstantial evidence suggests [21], then the electric vector position angles in Fig. 4 would have to be corrected by rotating them counter-clockwise/clockwise by $\sim 11^{\circ}$.

In the jet, the electric field vectors are oriented preferentially along its axis suggesting the presence of a shock at the interface between the ejected matter and the surrounding environment.

\section{Spectrum}

The global spectral index of Circinus X-1 between 4.8 and $8.6 \mathrm{GHz}$ remained virtually unchanged in the observing sessions between 1996-2006, at a mean value of $\alpha=-0.9 \pm 0.6\left(F_{v} \propto\right.$ $\left.v^{\alpha}\right)$. However, the spectrum flattens during outbursts (at periastron as well as at apastron), consistent both with a model in which the synchrotron radiation is produced in a cloud of relativistic particles accelerated by a shock front expanding adiabatically [22, 23], and with the internal shock model, in which the particles are accelerated in successive shocks produced in a quasi-continuous jet [24]. Our data set do not clearly rule out any of the two. The general shape of the spectrum between the two frequencies suggests an optically thin emitting region and strongly points towards a synchrotron origin for the radiation.

\section{Conclusions}

We have observed Circinus X-1 for almost 10 years between 1996-2006 at 4.8 and $8.6 \mathrm{GHz}$ using ATCA. The data reveals changes in the morphology and brightness of the system at timescales of days. On the other hand, the polarisation properties and the shape of the spectrum remain relatively stable during the period of the monitoring. The data is compatible with the presence in 
the system of a relativistic flow. The conservative upper limit to the proper motion obtained from the radio data cannot by itself rule out the "runaway hypothesis".

\section{Acknowledgments}

The Australia Telescope is funded by the Commonwealth of Australia for operation as a national facility managed by CSIRO. The X-ray data was provided by the ASM/RXTE teams at MIT and at the RXTE SOF and GOF at NASA's GSFC.

\section{References}

[1] Tennant A.F., Fabian A.C., Shafer R.A., 1986a, MNRAS, 219, 871

[2] Tennant A.F., Fabian A.C., Shafer R.A., 1986b, MNRAS, 221, 27

[3] Boutloukos S., van der Klis M., Altamirano D., Klein-Wolt M., Wijnands R., Jonker P.G., Fender R.P., 2006, ApJ, 653, 1435

[4] Kaluzienski L.J., Holt S.S., Boldt E.A., Serlemitsos P.J., 1976, ApJ, 208, L71

[5] Glass I.S., 1978, MNRAS, 183, 335

[6] Glass I.S., 1994, MNRAS, 268, 742

[7] Whelan J.A.J., Mayo S.K., Wickramasinghe D.T., Murdin P.G., Peterson B.A., Hawarden T.G., Longmore A.J., Haynes R.F., Goss W.M., Simons L.W., Caswell J.L., Little A.G., McAdam W.B., 1977, MNRAS, 181, 259

[8] Haynes R.F., Jauncey D.L., Murdin P.G., Goss W.M., Longmore A.J., Simons L.W., Milne D.K., Skellern D.J., 1978, MNRAS, 185, 661

[9] Haynes R.F., Komesaroff M.M., Little A.G., Jauncey D.L., Caswell J.L., Milne D.K., Kesteven M.J., Wellington K.J., Preston R.A., 1986, Nature, 324, 233

[10] Stewart R.T., Caswell J.L., Haynes R.F., Nelson G.J., 1993, MNRAS, 261, 593

[11] Heinz S., 2002, A\&A, 388, L40

[12] Tudose V., Fender R.P., Kaiser C.R., Tzioumis A.K., van der Klis M., Spencer, R.E., 2006, MNRAS, 372,417

[13] Heinz S., Schulz N.S., Brandt W.N., Galloway D.K., 2007, ApJ, 663, L93

[14] Fender R., Wu K., Johnston H., Tzioumis T., Jonker P., Spencer R., van der Klis M., 2004, Nature, 427, 222

[15] Clark D.H., Parkinson J.H., Caswell J.L., 1975, Nature, 254, 674

[16] Mignani R.P., De Luca A., Caraveo P.A., Mirabel I.F., 2002, A\&A, 386, 487

[17] Fender R., Spencer R., Tzioumis T., Wu K., van der Klis M., van Paradijs J., Johnston H., 1998, ApJ, 506, L121

[18] Fender R., Tzioumis T., Tudose V., 2005, Astron. Tel., 563, 1

[19] Nicolson G.D., 2007, Astron. Tel., 985, 1 
[20] Phillips C.J., Deller A., Amy S.W., Tingay S.J., Tzioumis A.K., Reynolds J.E., Jauncey D.L., Stevens J., Ellingsen S.P., Dickey J., Fender R.P., Tudose V., Nicolson G.D., 2007, MNRAS, 380, L11

[21] Johnston-Hollitt M., Hollitt C.P., Ekers R.D., 2004, in The Magnetized Interstellar Medium, eds. B. Uyaniker, W. Reich, and R. Wielebinski

[22] van der Laan H., 1966, Nature, 211, 1131

[23] Haynes R.F., Lerche I., Murdin P., 1980, A\&A, 87, 299

[24] Kaiser C.R., Sunyaev R., Spruit H.C., 2000, A\&A, 356, 975 